\title{
3D printing technology in percutaneous nephrolithotomy: babyhood but will be promising
}

\author{
Weizhou Wu ${ }^{1} \cdot$ Zhijian Zhao $^{1} \cdot$ Shike Zhang ${ }^{1} \cdot$ WenQi Wu ${ }^{1}$ (C)
}

Received: 16 December 2019 / Accepted: 21 December 2019 / Published online: 3 January 2020

c) Springer-Verlag GmbH Germany, part of Springer Nature 2020

\section{Dear Editor,}

With great interest, I read the article by $\mathrm{Xu}$ et al. [1]. The study showed that the 3D technique can reconstruct both the renal collecting system and urinary tract stones, as well as spines and ribs based on the CT scans, which could help to decide the most optimal calyx for puncture during percutaneous nephrolithotomy (PCNL) in vitro simulation. Their results also showed an enhanced stone clearance using the technique in 12 patients with staghorn stones.

Some matters are still worth considering. First, the distribution of renal segment arteries and interlobular arteries surrounding calyx was failed to be displayed on 3D printing models. As we know, vascular injury along the puncture access route is the most direct and vital reason for bleeding during PCNL. Moreover, clinical experience is another factor closely related to bleeding [2] and current studies suggest that puncture of the calyx fornix can reduce intraoperative bleeding and other complications during PCNL. Therefore, even the calyx for puncture has been determined, 3D printing technology has not yet been able to help to achieve calyx fornix puncture when facing the anatomical structures in reality, especially for junior surgeons.

Second, the stone-free rate of PCNL for simple kidney stones can be up to $96.9 \%$ [3]. Removing simple stones with the help of models is surely using a sledgehammer to crack a nut. To summarize, this technology is better suited for experienced urologists to deal with complex kidney stones, which will maximize the benefit of patients.

Finally, a more accurate 3D model will promote the 3D printing technology and benefit the operation. The segmentation using for 3D model is usually disposed by radiologists

WenQi Wu

wwqwml@163.com

1 Guangdong Key Laboratory of Urology, Department of Urology, Minimally Invasive Surgery Center, Guangzhou Institute of Urology, The First Affiliated Hospital of Guangzhou Medical University, Kangda Road 1\#, Haizhu District, Guangzhou 510230, Guangdong, China and its outcome of the model based on CT data could be affected by many subjective factors such as the selection of structure, imaging mode and the contrast-to-noise ratio. Also utilizing different attenuation thresholds to segment tissue from a CT image can yield not only different dimensions but also different shapes of 3D models, which has an impact on the ultimate effect of the models [4]. Thus, the construction of 3D model should be under the guidance of experienced radiologists to guarantee the accuracy.

We agree with the authors that 3D printing technology has its own broad prospects and operability among the treatment of renal staghron stones. This technology is potentially useful in the preoperative planning and in favor of shortening the learning curve of PCNL. We hope more high-quality trials to confirm the feasibility of 3D printing technology in PCNL.

\section{Compliance with ethical standards}

Conflict of interest The authors declare that they have no conflict of interest.

\section{References}

1. Xu Y, Yuan Y, Cai Y et al (2019) Use 3D printing technology to enhance stone free rate in single tract percutaneous nephrolithotomy for the treatment of staghorn stones. Urolithiasis. https ://doi.org/10.1007/s00240-019-01164-8

2. El-Nahas AR, Shokeir AA, El-Assmy AM et al (2007) Post-percutaneous nephrolithotomy extensive hemorrhage: a study of risk factors. J Urol 177(2):576-579

3. Abdelhafez MF, Bedke J, Amend B et al (2012) Minimally invasive percutaneous nephrolitholapaxy (PCNL) as an effective and safe procedure for large renal stones. BJU Int $110(11 \mathrm{Pt}$ C):E1022-1026

4. George E, Liacouras P, Rybicki FJ et al (2017) Measuring and establishing the accuracy and reproducibility of 3D printed medical models. Radiographics 37(5):1424-1450

Publisher's Note Springer Nature remains neutral with regard to jurisdictional claims in published maps and institutional affiliations. 